\title{
Calibration of GRACE Accelerometers Using Two Types of Reference Accelerations
}

\author{
Igor Koch, Akbar Shabanloui, and Jakob Flury
}

\begin{abstract}
Two approaches for the calibration of GRACE (Gravity Recovery And Climate Experiment) accelerometers are revisited. In the first approach, surface forces acting on the satellite are considered to derive the reference acceleration. In the second approach, the total acceleration consisting of a gravitational and a non-gravitational contribution is first determined from the reduced-dynamic orbits. The approximation of discrete satellite positions by a polynomial function allows the total acceleration to be obtained by a twofold derivative w.r.t. time. Calibration parameters (scale factor and bias) and statistical values are estimated for periods with a low and high solar activity. The quality of these two approaches shows dependencies on solar activity and consequent variations in the magnitude of the non-gravitational reference acceleration. Besides, the quality of the presented results is affected by the orientation of the orbital plane w.r.t. the Sun. The second approach is vitiated by a periodic disturbing signal on cross-track axis. This signal has been pointed out in earlier studies (Calabia et al., Aerosp Sci Technol 45, 2015; Calabia and Jin, Aerosp Sci Technol 49, 2016). We apply a moving window median filter to recover the underlying non-gravitational signal for accelerometer calibration. The calibration is accomplished by a direct comparison of reference accelerations and observed accelerometer measurements without introducing any a priori values or constraints. The focus of this work is more sensor oriented than gravity field recovery (GFR) related. Nevertheless, the results can be used as initial values for precise orbit determination (POD) or for pre-processing of accelerometer measurements in a multi step gravity field recovery approach (Klinger and Mayer-Gürr, Adv Space Res 58(9), 2016).
\end{abstract}

\section{Keywords}

Accelerometry $\cdot$ GRACE $\cdot$ Satellite accelerometer calibration

\section{$1 \quad$ Introduction}

The GRACE mission has been in orbit since 2002 and is based on two twin satellites, GRACE A and B, that observe static and temporal changes of Earth's gravity field

I. Koch $(\bowtie) \cdot$ A. Shabanloui $\cdot$ J. Flury

Institut für Erdmessung, Leibniz Universität Hannover, Hannover, Germany

e-mail: koch@ife.uni-hannover.de
(Tapley et al. 2004). For this purpose, the knowledge of the non-gravitational forces acting on the satellites is of major importance, since for the recovery of Earth's gravity field parameters, acceleration effects of gravitational and non-gravitational nature have to be separated. Therefore, an accelerometer that measures the total non-gravitational acceleration experienced by the satellite is located in the center of mass of each satellite (Flury et al. 2008). However, these measurements are not absolute values, but rather have to be corrected in their magnitude and variational amplitude 
by a calibration in the post-processing. In the scope of gravity field estimation several methods to calibrate accelerometer measurements are applied. Usually, calibration parameters are estimated directly within precise orbit determination or gravity field recovery procedures, e.g. Van Helleputte et al. (2009), Dahle et al. (2013), Watkins and Yuan (2014), Bettadpur (2018). There exist a priori calibration parameters based on GRACE data between the start of the mission and end of March 2009, but because of possible variations due to the specific analysis of the data, these parameters should only be regarded as recommendations (Bettadpur 2009).

It should be noted that a calibration parameter estimation within POD or GFR is not part of this work. Here the focus is laid on constraint-free estimation of non-gravitational acceleration that is used as reference for the calibration of accelerometer data. Two approaches for the computation of the non-gravitational reference acceleration are realized. In the first approach, further denoted as NGM (nongravitational modeling), surface accelerations acting on a spacecraft composed of atmospheric drag and direct solar radiation pressure are taken into account. This approach can be regarded as a basic method for the estimation of non-gravitational reference acceleration. For instance, a twostep calibration process carried out in Klinger and MayerGürr (2016) uses approach NGM for initial accelerometer calibration. The modeled reference acceleration enables a threshold-based screening of the accelerometer data, while in a second step the pre-screened accelerometer data is used within GFR, where the calibration parameters are reestimated.

In the second approach, the total acceleration consisting of a gravitational and non-gravitational contribution is first determined from the GNSS-based reduced-dynamic orbits. The approximation of discrete satellite positions by a polynomial function allows the total acceleration to be obtained by a twofold derivative w.r.t. time. Subtraction of the gravitational part provides a further reference, onwards referred as NGO (Non-Gravitational acceleration based on Orbit derivatives), for accelerometer calibration. By applying different strategies for numerical differentiation of geometric and reduceddynamic orbit products, this approach has been presented in previous studies, e.g. Bezděk (2010) and Calabia et al. (2015).

The main focus of this work is the comparison of these two approaches w.r.t. different strengths of solar activity. Therefore, accelerometer calibration parameters, i.e. scale factor and bias, and corresponding statistical values for two periods, one with a high solar activity as $\mathrm{P}_{\max }$ covering from $2002 / 11 / 01$ to $2003 / 01 / 31$ and the other with a low solar activity $\mathrm{P}_{\min }$ covering from 2008/06/01 to 2008/08/31 (cf. Fig. 1), are estimated and investigated.

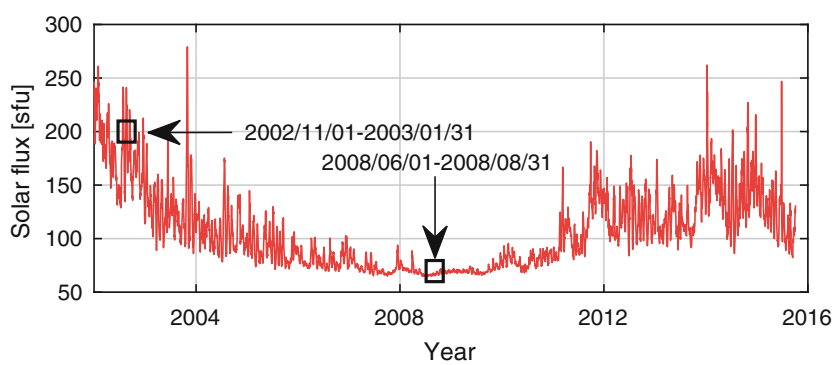

Fig. 1 Solar activity during the period 2002-2016. The two periods studied in this work are highlighted

Table 1 GRACE Level-1B products used in this study

\begin{tabular}{ll}
\hline Product & Data \\
\hline GNV1B & Reduced-dynamic position, velocity \\
\hline ACC1B & Linear acceleration \\
\hline SCA1B & Satellite attitude (quaternions) \\
\hline MAS1B & Satellite mass \\
\hline THR1B & Thruster events \\
\hline
\end{tabular}

\section{GRACE Data}

In this study several GRACE Level-1B (L1B) data products are used (Case et al. 2010). An overview is given in Table 1. GNV1B products contain satellite positions and velocities at $5 \mathrm{~s}$ interval obtained from reduced-dynamic POD. Linear accelerations in the GRACE science reference frame (SRF) are part of ACC1B data products. These measurements are given every second. Quaternions from SCA1B are utilized to transform between inertial and orbital frame. Furthermore, the mass of the satellite from MAS1B data products is essential for the calculation of the non-gravitational acceleration effects. Here the daily mean mass is utilized. Thruster firing events from THR1B are used to filter thruster affected accelerometer measurements.

\section{$3 \quad$ Methods}

\subsection{NGM Approach}

In the approach NGM, the reference acceleration $\mathbf{a}_{\text {ref,NGM }}$ is computed as the sum of modeled non-gravitational effects $\mathbf{a}_{\mathrm{ngr}, i}$ :

$$
\mathbf{a}_{\mathrm{ref}, \mathrm{NGM}}=\sum_{i=1}^{2} \mathbf{a}_{\mathrm{ngr}, i} .
$$

Non-gravitational acceleration models and parameters that are used to determine the sum of separate acceleration effects $\mathbf{a}_{\mathrm{ngr}, i}$ are summarized in the upper part of Table 2. Compared to atmospheric drag and solar radiation pressure, the 
Table 2 Overview of the used non-gravitational and gravitational models and parameters

\begin{tabular}{|c|c|c|c|c|}
\hline & $i$ & Acceleration & Model: Parameters & Reference \\
\hline \multirow{9}{*}{ 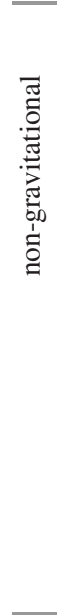 } & \multirow[t]{4}{*}{1} & \multirow[t]{4}{*}{ Atmospheric drag } & $\begin{array}{l}\text { Atmospheric density model: } \\
\text { NRLMSISE-00 }\end{array}$ & Picone et al. (2002) \\
\hline & & & Wind: Earth's atmosphere co-rotation & $\begin{array}{l}\text { Montenbruck and Gill } \\
(2005)\end{array}$ \\
\hline & & & $\begin{array}{l}\text { Drag coefficient: } C_{\mathrm{D}}=3.5\left(\mathrm{P}_{\max }\right), \\
C_{\mathrm{D}}=2.3\left(\mathrm{P}_{\min }\right)\end{array}$ & \\
\hline & & & Satellite macro model & Bettadpur (2012) \\
\hline & \multirow[t]{5}{*}{2} & \multirow[t]{5}{*}{ Solar radiation pressure } & Shadow model: conic & $\begin{array}{l}\text { Montenbruck and Gill } \\
\text { (2005) }\end{array}$ \\
\hline & & & Solar flux at $1 \mathrm{AU}: W=1367 \mathrm{Watt} / \mathrm{m}^{2}$ & $\begin{array}{l}\text { Montenbruck and Gill } \\
(2005)\end{array}$ \\
\hline & & & Solar ephemerides: DE430 & Folkner et al. (2014) \\
\hline & & & $\begin{array}{l}\text { Solar radiation pressure coefficient: } \\
C_{\mathrm{R}}=1.1\left(\mathrm{P}_{\max }\right), C_{\mathrm{R}}=1.0\left(\mathrm{P}_{\min }\right)\end{array}$ & \\
\hline & & & Satellite macro model & Bettadpur (2012) \\
\hline \multirow{10}{*}{. } & 1 & Static gravity & EIGEN-6S4, $C_{20}$ : tide free, $n_{\max }=180$ & Förste et al. (2015) \\
\hline & \multirow[t]{2}{*}{2} & \multirow[t]{2}{*}{ Temporal gravity } & $\begin{array}{l}\text { Long wavelength: EIGEN-6S4, drift, } \\
\text { annual and semi-annual variations }\end{array}$ & Förste et al. (2015) \\
\hline & & & Short wavelength: AOD1B RL06 & Dobslaw et al. (2017) \\
\hline & 3 & Third bodies & $\begin{array}{l}\text { Ephemerides: DE430 including Sun and } \\
\text { Moon }\end{array}$ & Folkner et al. (2014) \\
\hline & 4 & Solid Earth tides & $\begin{array}{l}\text { Ephemerides: DE } 430 \text { including Sun and } \\
\text { Moon }\end{array}$ & Folkner et al. (2014) \\
\hline & 5 & Ocean tides & $\begin{array}{l}\text { EOT11a, } n_{\max }=120, \text { admittance theory } \\
\text { for minor waves }\end{array}$ & Rieser et al. (2012) \\
\hline & 6 & Relativistic effects & Schwarzschild effect & Petit and Luzum (2010) \\
\hline & 7 & Pole tides & IERS Conventions 2010 & Petit and Luzum (2010) \\
\hline & 8 & Ocean pole tides & IERS Conventions 2010 & $\begin{array}{l}\text { Petit and Luzum (2010) } \\
\text { Desai (2002) }\end{array}$ \\
\hline & 9 & Atmospheric tides & N1 model, seasonal means & $\begin{array}{l}\text { Biancale and Bode } \\
(2006)\end{array}$ \\
\hline
\end{tabular}

corresponding magnitude of these effects is usually several orders smaller. Therefore, the acceleration caused by albedo and thermal infrared is not taken into account in this study. Nevertheless, the influence of these effects will be studied in future articles.

As a common inertial reference system the Geocentric Celestial Reference System (GCRS) is used. The transformation of satellite GNV1B positions that are provided in the International Terrestrial Reference System (ITRS) is done according to Petit and Luzum (2010).

\subsection{NGO Approach}

In the approach NGO, the non-gravitational reference acceleration $\mathbf{a}_{\text {ref,NGO is determined by the difference of the total }}$ acceleration $\mathbf{a}$ and the sum of gravitational acceleration effects $\mathbf{a}_{\mathrm{gr}, i}$ :

$$
\mathbf{a}_{\mathrm{ref}, \mathrm{NGO}}=\mathbf{a}-\sum_{i=1}^{9} \mathbf{a}_{\mathrm{gr}, i} \text {. }
$$

Gravitational background models and parameters used for predicting the sum of acceleration effects $\mathbf{a}_{\mathrm{gr}, i}$ can be found in the bottom part of Table 2. The total acceleration of a satellite $\mathbf{a}$ is obtained by taking the second derivative of the GNV1B positions w.r.t. time. In this scheme, after transformation of the positions to GCRS, the smoothing polynomial approach is used to calculate piece-wise polynomial coefficients based on least squares adjustment (Luers and Wenning 1971). In its general form, a polynomial function of degree $n$ can be described by Eq. (3):

$$
f(\tau)=\sum_{i=0}^{n} a_{i} \tau^{i}
$$

In this equation $a_{i}$ denote polynomial coefficients and the argument $\tau$ corresponds to the time of the sample points. The parameter vector $\mathbf{x}$ containing the piece-wise polynomial coefficients is estimated by least squares adjustment:

$$
\mathbf{x}=\left(\mathbf{A}^{\mathrm{T}} \mathbf{A}\right)^{-1} \mathbf{A}^{\mathrm{T}} \mathbf{l}
$$


Here, $\mathbf{I}$ denotes the observation vector which includes the $x$-, $y$ - or $z$-components of satellite's inertial positions and $\mathbf{A}$ is the corresponding design matrix. For the computation of the polynomial coefficients a unit weight matrix is employed. In this work, a polynomial of degree $n=7$ has been fitted to $m=21$ position components. Note that the time arguments $\tau_{1} \ldots \tau_{m}$ of the regarded position components were normalized to $[-1,1]$ in order to make the estimation of the polynomial coefficients numerically stable. This method takes advantage of the equidistant $5 \mathrm{~s}$ GNV1B positions, so $\tau=0$, assumed that $m$ is uneven, always corresponds to the sample point in the middle of the interval $[-1,1]$ and that is also the sample point whose acceleration is evaluated.

\subsection{Accelerometer Calibration}

The magnitude of the ACC1B measurements $\mathbf{a}_{\text {uncal }}$ is corrected by the introduction of a bias vector $\mathbf{b}$. The amplitude is adjusted by a diagonal scale matrix $\mathbf{S}$ containing a scale factor for each axis:

$$
\mathbf{a}_{\mathrm{cal}}=\mathbf{S} \mathbf{a}_{\mathrm{uncal}}+\mathbf{b}
$$

The unknown scale matrices and bias vectors are determined on a daily basis using least squares adjustment. Note that all the terms in Eq. (5) refer to the GRACE science reference frame. In order to use the computed reference accelerations (cf. Eqs. (1) and (2)) as observations in least squares adjustment, the computed reference accelerations have to be transformed from GCRS to SRF. For this purpose, SCA1B quaternions are used to form the corresponding rotation matrices as described in Sutton (2008). Accelerometer measurements that are affected by thruster firings are removed before adjustment. In order to exclude spike affected accelerometer measurements from the adjustment, values outside the 3sigma distribution are not considered. Although the reduceddynamic approach acts as a low-pass filter during orbit determination, generated positions contain noise that is amplified during differentiation considerably. In order to decrease the noisy characteristic of the accelerations obtained with approach NGO before calibration parameter estimation, a moving window median filter with a size of 30 acceleration measurements is applied.

\section{$4 \quad$ Results and Discussion}

In general, different parameters, variables and assumptions affect the quality of the reference accelerations. Particularly, the approach NGM is based on numerous parameters and models that are often inaccurate and biased. Some of these parameters are for example the atmospheric density and wind model, solar flux and geomagnetic proxies, eclipse modeling, the macro model and variables such as the drag and solar radiation pressure coefficients (Doornbos 2011). In POD, the unknown parameters are estimated in order to absorb biases in the models and noise in the measurements. Besides, general quality of approach NGO is primary affected by choice and actuality of employed models (Bezděk 2010). In this study, extreme solar condition periods are regarded. The quality of the results is clearly dependent on the magnitude of the non-gravitational acceleration that is affected by solar activity. The F10.7 solar radio flux during period $\mathrm{P}_{\max }$ fluctuates between 114 and 213 solar flux units (sfu) (cf. Fig. 1) which corresponds to a rather high solar activity. The solar activity during period $\mathrm{P}_{\min }$ varies between 64 and 68 sfu, so this period can be regarded as the least sun-disturbed period during GRACE operating time. These variations in solar activity lead to differences in the magnitude of the total non-gravitational reference accelerations.

To get an impression how the $\mathrm{ACC} 1 \mathrm{~B}$ measurements and the computed reference accelerations using the two approaches fit together, some calibrated datasets are illustrated in Fig. 2. The similarity of the calibrated ACC1B acceleration with the respective reference acceleration has been quantified by means of the correlation coefficient. The monthly mean correlation coefficients are shown in Table 3. It is striking that the variability of NGM related to the investigated periods is, in contrast to approach NGO, minimal. The correlation coefficients for NGM range from 0.91 to 0.95 , while for approach $\mathrm{NGO}$, a strong variance can be observed that is dependent on the magnitude of the computed reference accelerations. Note that the presented correlation coefficients are not the best quality indicators for the calibration parameters, especially the biases, but good indicators to assess the quality of the recovered nongravitational signal using approach NGO.

An aspect that determines the quality of the constraintfree and daily calibration parameter estimation, is the orientation of the satellite's orbital plane w.r.t. the Sun, often referred to as $\beta^{\prime}$ angle. $\beta^{\prime}$ equals $0^{\circ}$ when the satellite's orbital plane coincidences with the Earth-Sun line of sight. If the orbital plane is perpendicular w.r.t. the Earth-Sun line of sight, $\beta^{\prime}$ equals $\pm 90^{\circ}$. The drift of the GRACE orbit's ascending node prevents $\beta^{\prime}$ to reach $\pm 90^{\circ}$. In this work, $\beta^{\prime}$ reaches $+71^{\circ}$ and $-73^{\circ}$ for periods $\mathrm{P}_{\max }$ and $\mathrm{P}_{\text {min }}$ respectively. It was decided to exclude the epochs with aforementioned maximum/minimum $\beta^{\prime} \pm 7^{\circ}$, since during this periods unrealistic calibration parameters and small correlation coefficients were obtained. This period was neither considered for the calculation of the correlation coefficients that are shown in Table 3, nor for the mean calibration parameters that will be presented later in this chapter. The two excluded periods are highlighted in Fig. 3. 


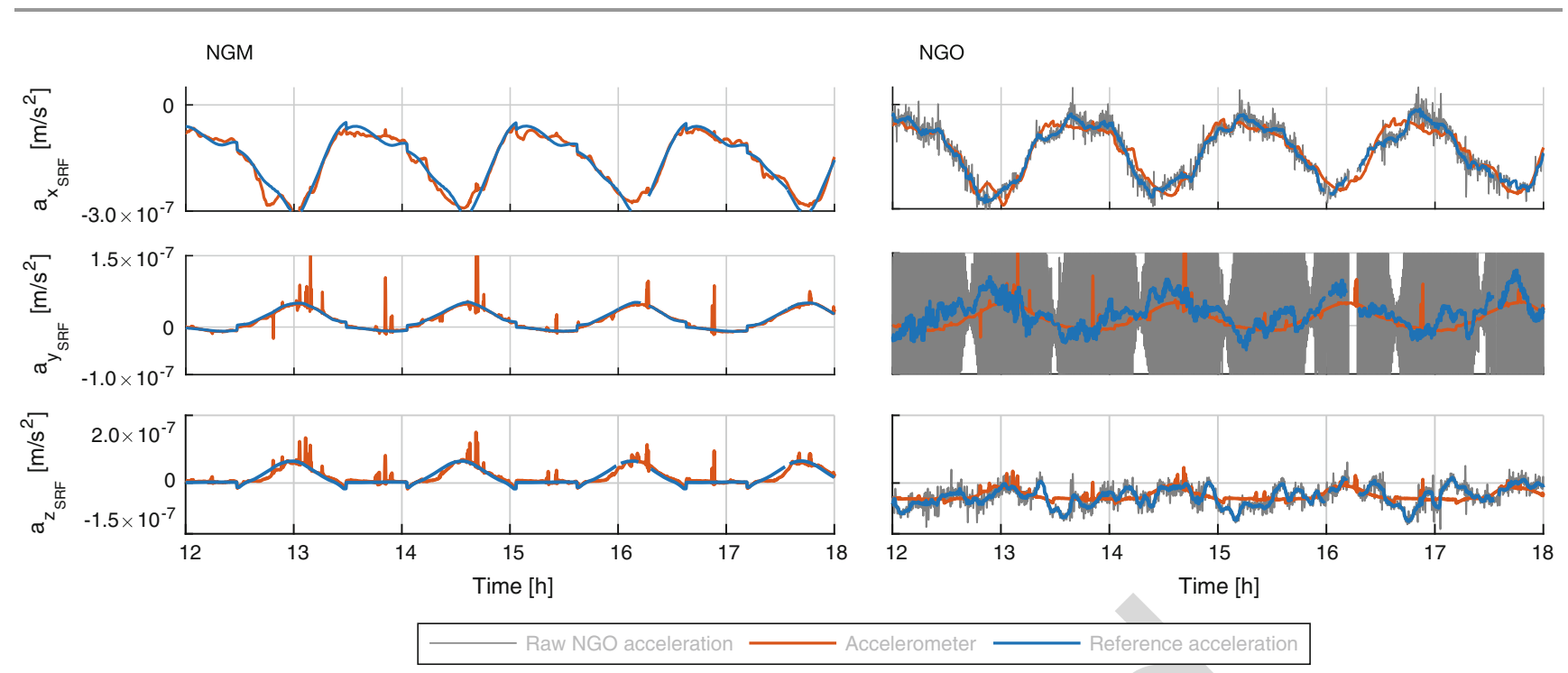

Fig. 2 Exemplary calibrated GRACE B ACC1B accelerometer measurements (red) using approaches NGM (left) and NGO (right) for the epoch 2003/01/01. Upper panel: $x_{\mathrm{SRF}}$, middle panel: $y_{\mathrm{SRF}}$ and bottom panel: $z_{\mathrm{SRF}}$

Table 3 Mean correlation coefficient of calibrated ACC1B data and modeled reference acceleration

\begin{tabular}{llllll}
\hline & $\mathrm{P}_{\max }$ & & & $\mathrm{P}_{\min }$ & \\
\cline { 2 - 3 } & NGM & NGO & & NGM & NGO \\
\hline$x_{\text {SRF }}$ & 0.93 & 0.96 & & 0.93 & 0.77 \\
\hline$y_{\text {SRF }}$ & 0.95 & 0.68 & & 0.91 & 0.52 \\
\hline$z_{\text {SRF }}$ & 0.91 & 0.51 & & 0.93 & 0.11 \\
\hline
\end{tabular}

An aspect important to consider when applying approach NGO to the GNV1B reduced-dynamic orbit data, is a disturbing signal of unknown origin in $y_{\mathrm{SRF}}$ direction, with an absolute amplitude of about $1 \times 10^{-6} \mathrm{~m} / \mathrm{s}^{2}$ and a dominant period of half a revolution. This kind of disturbing signal has already been pointed out in Calabia et al. (2015) and Calabia and Jin (2016) for different periods of time. In Calabia and Jin (2016) the authors indicate a maximum amplitude of $6 \times 10^{-6} \mathrm{~m} / \mathrm{s}^{2}$ for $y_{\mathrm{SRF}}$ and $1.5 \times 10^{-6} \mathrm{~m} / \mathrm{s}^{2}$ for $z_{\mathrm{SRF}}$ direction. In Calabia et al. (2015) the authors refer to Bezděk (2010), where the $y_{\mathrm{SRF}}$ axis seems to be affected by a similar periodic disturbing signal. By using the aforementioned moving window median filter an underlying signal that is suitable for calibration can be retrieved for period $\mathrm{P}_{\max }$ (cf. Fig. 2). The recovered signal is in good agreement with the accelerometer measurements. The corresponding mean correlation coefficient is 0.68 (cf. Table 3 ). In contrast, during weak solar activity the correlation coefficient of the recovered reference acceleration w.r.t. the calibrated accelerometer measurements is only 0.52 .

A time series of estimated daily calibration parameters for the two investigated periods can be seen in Fig. 3. In addition, long term fits to estimated biases from POD are plotted as a reference (Bettadpur 2009). Mean estimated calibration parameters and corresponding standard deviations are reported in Table 4. A comparison of the reference values with the obtained daily calibration parameters should be treated warily because different types of approaches are compared. In this study, assumptions, i.e. that the scale factors are approximately one over the whole period, are not introduced. Although, for $x_{\mathrm{SRF}}$ often realistic values can be obtained, daily scales for $y_{\mathrm{SRF}}$ and $z_{\mathrm{SRF}}$ usually vary a lot, i.e. Klinger and Mayer-Gürr (2016) and Bruinsma et al. (2007). Using the two presented approaches, the daily scale factors and biases are optimal in a least squares sense w.r.t. the modeled reference accelerations and are not affected by any constraints. Despite the mentioned variance of the calibration parameters, the presented calibration procedure may lead to highly correlated calibration parameters (Bruinsma et al. 2007; Van Helleputte et al. 2009). Although out of the scope of this study and objective of further investigations, constraints can be applied to de-correlate scale factors and biases, i.e. Bruinsma et al. (2007), Van Helleputte et al. (2009). The long term fits from Bettadpur (2009) are a generalized form of calibration parameters obtained in POD. In POD and GFR these values can be treated as initial values and corrections to these values have to be estimated iteratively.

In Fig. 3 and Table 4 it can be seen that approach NGO differs from NGM when estimating parameters for the $x_{\mathrm{SRF}}$ component for period $\mathrm{P}_{\max }$. The NGO bias time series shows a stable behavior over the time span of three months and is in good agreement with the long term biases. Similar aspects can be observed for the $x_{\mathrm{SRF}}$ scale. Although realistic values are obtained for both approaches (NGM: 0.91, NGO: 0.93), the standard deviation of approach NGO is more than three times smaller. To obtain a mean scale value comparable to 


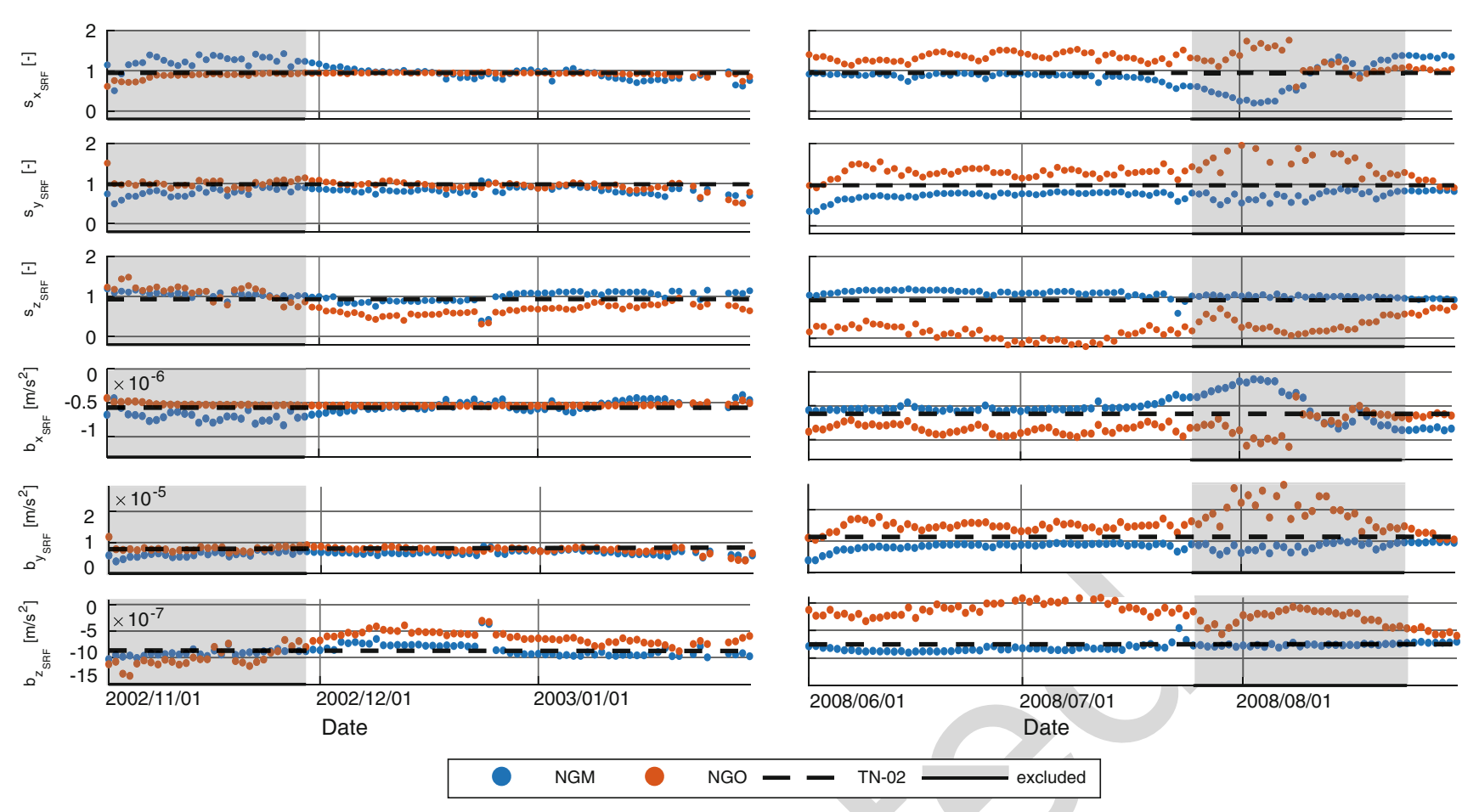

Fig. 3 Daily estimated accelerometer calibration parameters: Left ( $\left.\mathrm{P}_{\max }: 2002 / 11 / 01-2003 / 01 / 31\right)$; Right $\left(\mathrm{P}_{\min }\right.$ : 2008/06/01-2008/08/31). First three upper rows represent scale parameters and the last three rows show bias parameters

Table 4 Mean accelerometer calibration parameters for the period with a strong solar activity $\left(\mathrm{P}_{\max }\right.$ : 2002/11/01-2003/01/31) and weak solar activity $\left(\mathrm{P}_{\min }\right.$ : 2008/06/01-2008/08/31) using approaches NGM and NGO

\begin{tabular}{|c|c|c|c|c|c|c|c|c|c|}
\hline & & \multicolumn{4}{|l|}{$\mathrm{P}_{\max }$} & \multicolumn{4}{|l|}{$\mathrm{P}_{\min }$} \\
\hline & & \multicolumn{2}{|c|}{ Scale $[-]$} & \multicolumn{2}{|l|}{$\operatorname{Bias}\left[\mathrm{m} / \mathrm{s}^{2}\right]$} & \multicolumn{2}{|c|}{ Scale $[-]$} & \multicolumn{2}{|l|}{$\operatorname{Bias}\left[\mathrm{m} / \mathrm{s}^{2}\right]$} \\
\hline & & NGM & NGO & NGM & NGO & NGM & NGO & NGM & $\mathrm{NGO}$ \\
\hline \multirow[t]{2}{*}{$x_{\mathrm{SRF}}$} & Mean & 0.91 & 0.93 & $-5.38 \times 10^{-7}$ & $-5.35 \times 10^{-7}$ & 0.92 & 1.31 & $-5.69 \times 10^{-7}$ & $-8.22 \times 10^{-7}$ \\
\hline & $\overline{\text { Std }}$ & 0.13 & 0.04 & $6.79 \times 10^{-8}$ & $1.42 \times 10^{-8}$ & 0.18 & 0.14 & $1.12 \times 10^{-7}$ & $9.04 \times 10^{-8}$ \\
\hline \multirow[t]{2}{*}{$y_{\mathrm{SRF}}$} & Mean & 0.83 & 0.93 & $6.72 \times 10^{-6}$ & $7.57 \times 10^{-6}$ & 0.74 & 1.26 & $8.45 \times 10^{-6}$ & $1.44 \times 10^{-5}$ \\
\hline & $\overline{\text { Std }}$ & 0.09 & 0.12 & $7.30 \times 10^{-7}$ & $9.18 \times 10^{-7}$ & 0.11 & 0.15 & $1.22 \times 10^{-6}$ & $1.67 \times 10^{-6}$ \\
\hline \multirow[t]{2}{*}{$z_{\mathrm{SRF}}$} & Mean & 0.98 & 0.66 & $-8.46 \times 10^{-7}$ & $-6.15 \times 10^{-7}$ & 1.09 & 0.18 & $-8.14 \times 10^{-7}$ & $-1.45 \times 10^{-7}$ \\
\hline & $\overline{\text { Std }}$ & 0.15 & 0.13 & $1.29 \times 10^{-7}$ & $1.13 \times 10^{-7}$ & 0.11 & 0.24 & $7.30 \times 10^{-8}$ & $1.82 \times 10^{-7}$ \\
\hline
\end{tabular}

the value derived from approach $\mathrm{NGO}$, the drag coefficient during period $\mathrm{P}_{\max }$ has to be set rather large (cf. Table 2). It is important to note that the strong variability of the solar flux during period $\mathrm{P}_{\max }$ demands the employment of a variable drag coefficient for approach NGM instead of a constant coefficient. This means that external information from POD could help to decrease the standard deviation of the calibration parameters obtained with approach NGM. For $y_{\mathrm{SRF}}$ and especially $z_{\mathrm{SRF}}$ directions, bigger disagreements between the two approaches can be seen. In comparison to the constant scale and long term bias, there is a better agreement for $y_{\mathrm{SRF}}$ when using approach NGO. In contrast, for $z_{\mathrm{SRF}}$ more realistic values are obtained using approach NGM. Because of the weak signal in $z_{\mathrm{SRF}}$ and the amplification of noise, it is more difficult to obtain appropriate scale factors and biases with approach NGO.
Daily calibration parameters for period $\mathrm{P}_{\text {min }}$ are shown on the right side of Fig. 3. During small solar activity approach NGM differs from approach NGO considerably. Compared to approach NGO, obtained calibration parameters using approach NGM are closer to the constant scale factors and long term biases. Especially calibration parameters of the $x_{\mathrm{SRF}}$ and $z_{\mathrm{SRF}}$ components fit very well with the reference values reported in Bettadpur (2009). In comparison to period $\mathrm{P}_{\max }$, no meaningful calibration parameters were obtained using approach NGO. The small magnitude of the non-gravitational acceleration decreases the signal-to-noise ratio (SNR) of the non-gravitational accelerations extracted from the reduced-dynamic orbits and makes it impossible to obtain adequate calibration parameters without any constraints. Most notable deviations from the constant scales and long term biases around the $\beta^{\prime}$ turning point can be seen 
for $x_{\mathrm{SRF}}$ during period $\mathrm{P}_{\min }$. As the orbital plane reaches this orientation w.r.t. the Sun, the satellite spends minimal time in the Earth's shadow region. This mentioned aspect in combination with a very small solar activity leads to measured and modeled non-gravitational accelerations with minimal variation of the signal amplitude. This small signal variation causes highly correlated calibration parameters.

\section{Conclusions}

In this work we studied if two types of modeled accelerations can serve as a good reference for the calibration of accelerometer measurements. We solved for daily scale factors and biases without the introduction of any a priori values and constraints for two 90 day periods during strong and weak solar activities. The approach NGM is first of all dependent on a reasonable choice of the drag coefficient that suits the specific activity of the sun. In contrast, approach NGO needs the employment of accurate time-variable gravity models.

This study showed that the quality of approach NGM is not as much affected by the solar activity (and therefore the absolute magnitude of the modeled reference acceleration) as approach NGO. For approach NGO, realistic scales and biases only could be obtained for the $x_{\mathrm{SRF}}$ and $y_{\mathrm{SRF}}$ directions during the period with strong solar activity. The quality of the obtained daily calibration parameters for the period with low solar activity using approach NGO is highly affected by the decreased signal-to-noise ratio of the non-gravitational acceleration extracted from the reduced dynamic orbits. This makes it impossible to estimate adequate scale factors and biases at the same time. Nevertheless, we think that if we would only solve for the biases, better results would always be obtained with approach NGO which employs GNSS measurements and accurate time-variable gravity models. To solely solve for the biases guarantees that the results are not distorted by unrealistic scale factors.

Furthermore, we confirm that when applying approach NGO to the GNV1B orbits, a disturbing signal on $y_{\mathrm{SRF}}$ axis is present. We used a moving median filter to successfully recover the underlying signal. Although further investigations are needed, our first experiments using our own computed reduced-dynamic orbits for approach NGO, show that the disturbing signal is highly likely caused by the characteristics of the provided GNV1B orbit data.

Acknowledgements We are thankful for the valuable comments of the three anonymous reviewers who helped to improve this article considerably. JPL is acknowledged for providing GRACE Level 1B data. Collaborative Research Centre 1128 "Relativistic Geodesy and Gravimetry with Quantum Sensors (geo-Q)" is acknowledged for financial support.

\section{References}

Bettadpur S (2009) Recommendation for a-priori bias \& scale parameters for Level-1B ACC data (version 2). Tech. note, Center for Space Research

Bettadpur S (2012) Product specification document GRACE 327-720. Tech. rep., Center for Space Research

Bettadpur S (2018) UTCSR Level-2 processing standards document for Level-2 product release 0006. Tech. rep., Center for Space Research

Bezděk A (2010) Calibration of accelerometers aboard GRACE satellites by comparison with POD-based nongravitational accelerations. J Geodyn 50

Biancale R, Bode A (2006) Mean annual and seasonal atmospheric tide models based on 3-hourly and 6-hourly ECMWF surface pressure data. Scientific Technical Report STR06/01, GeoForschungsZentrum Potsdam

Bruinsma S, Biancale R, Perosanz F (2007) Calibration parameters of the CHAMP and GRACE accelerometers. Poster, CNES, department of Terrestrial and Planetary Geodesy, GeoForschungsZentrum Potsdam

Calabia A, Jin S (2016) Assessment of conservative force models from GRACE accelerometers and precise orbit determination. Aerosp Sci Technol 49

Calabia A, Jin S, Tenzer R (2015) A new GPS-based calibration of GRACE accelerometers using the arc-to-chord threshold uncovered sinusoidal disturbing signal. Aerosp Sci Technol 45

Case K, Kruizinga G, Wu SC (2010) GRACE Level 1B data product user handbook. Tech. rep., Jet Propulsion Laboratory

Dahle C, Flechtner F, Gruber C, König D, König R, Michalak G, Neumayer K-H (2012) GFZ GRACE Level-2 processing standards document for Level-2 product release 0005. Tech. rep., GeoForschungsZentrum

Desai S (2002) Observing the pole tide with satellite altimetry. J Geophys 1078(C11)

Dobslaw H, Bergmann-Wolf I, Dill R, Poropat L, Thomas M, Dahle C, Esselborn S, König R, Flechtner F (2017) A new high-resolution model of non-tidal atmosphere and ocean mass variability for dealiasing of satellite gravity observations: AOD1B RL06, 2017. Geophys J Int 211(1):263-269

Doornbos E (2011) Thermospheric density and wind determination from satellite dynamics. Ph.D thesis, Delft University of Technology

Flury J, Bettadpur S, Tapley BD (2008) Precise accelerometry onboard the GRACE gravity field satellite mission. Adv Space Res 42(8):1414-1423

Folkner WM, Williams JG, Boggs, DH, Park RS, Kuchynka P (2014) The planetary and lunar ephemerides DE430 and DE431. IPN Progress Report

Förste C, Bruinsma S, Rudenko S, Abrikosov O, Lemoine JM, Marty JC, Neumayer KH, Biancale R (2015) EIGEN-6S4: A time-variable satellite-only gravity field model to d/o 300 based on LAGEOS, GRACE and GOCE data from the collaboration of GFZ Potsdam and GRGS Toulouse. European Geosciences Union, General Assembly 2015 Vienna (Austria) 12-17 April 2015

Van Helleputte T, Doornbos E, Visser P (2009) CHAMP and GRACE accelerometer calibration by GPS-based orbit determination. Adv Space Res 43:1890-1896

Klinger B, Mayer-Gürr T (2016) The role of accelerometer data calibration within GRACE gravity field recovery: Results from ITSGGrace2016. Adv Space Res 58(9)

Luers JK, Wenning RH (1971) Polynomial smoothing: linear vs cubic. Technometrics 13(3):589-600

Montenbruck O, Gill E (2005) Satellite orbits - models, methods and applications. Springer, Berlin, Heidelberg 
Petit G, Luzum B (eds) (2010) IERS Conventions 2010. Verlag des Bundesamts für Kartographie und Geodäsie, Frankfurt am Main

Picone JM, Hedin AE, Drob DP (2002) NRLMSISE-00 empirical model of the atmosphere: Statistical comparisons and scientific issues. J Geophys Res 107

Rieser D, Mayer-Gürr T, Savcenko R, Bosch W, Wünsch J, Dahle C, Flechtner F (2012) The ocean tide model EOT11a in spherical harmonics representation. Tech. note
Sutton E (2008) Effects of solar disturbances on the thermosphere densities and winds from CHAMP and GRACE satellite accelerometer data. University of Colorado at Boulder

Tapley BD, Bettadpur S, Watkins M, Reigber C (2004) The gravity recovery and climate experiment: Mission overview and early results. Geophys Res Lett 31(9)

Watkins MM, Yuan D-N (2014) JPL Level-2 processing standards document, for Level-2 product release 05.1. Tech. rep., Jet Propulsion Laboratory 